\title{
Enhancement of prestack diffraction data and attributes using a traveltime decomposition approach
}

\author{
Alexander Bauer, Benjamin Schwarz and Dirk Gajewski \\ Institute of Geophysics \\ University of Hamburg \\ Bundesstraße 55 \\ D-20146 Hamburg (Germany)
}

(January 8, 2016)

Keywords: CRS, stacking, moveout, diffraction, finite-offset

\begin{abstract}
Diffractions not only carry important information about small-scale subsurface structures, they also possess unique properties, which make them a powerful tool for seismic processing and imaging. Since a diffractor scatters an incoming wave to all directions, a diffraction event implies better illumination than a reflection, because the rays travel through larger parts of the subsurface. Furthermore, unlike the reflection case, in which the emergence location of the reflected wave depends on the source position, in the diffraction case Snell's law does not hold and, thus, up-going and down-going raypaths are decoupled. Based on this decoupling, we motivate a diffraction traveltime decomposition principle, which establishes a direct connection between zero-offset and finite-offset diffraction wavefield attributes. By making use of this approach, we are able to enhance diffractions and obtain high-quality diffraction wavefield attributes at arbitrary offsets in the prestack domain solely based on zero-offset processing without any further optimization of attributes. We show the accuracy
\end{abstract}


of the method by fitting diffraction traveltimes and on simple waveform data. Application to complex synthetic data shows the ability of the proposed approach to enhance diffractions and provide high-quality wavefield attributes even in sparsely illuminated regions such as subsalt areas. The promising results reveal a high potential for improved prestack data enhancement and further applications such as efficient diffraction-based finite-offset tomography. 


\section{INTRODUCTION}

Conventional seismic processing techniques are designed to image and enhance reflection events. However, reflected waves are not suitable for high-resolution structural imaging of features below the Rayleigh limit of half a seismic wavelength (e.g. Dell and Gajewski, 2011). Information about these small-scale structures such as edges, faults, pinch-outs and small-size scattering objects is encoded in the diffraction response of the subsurface (Khaidukov et al., 2004; Fomel et al., 2007). Therefore, the imaging of diffracted waves is a crucial challenge in seismic processing. Recently, different workflows with the goal to separate diffractions from reflections and to enhance diffractions in the post-stack domain have been presented (Fomel et al., 2007; Berkovitch et al., 2009; Dell and Gajewski, 2011). However, an important goal of diffraction imaging is the separation of diffractions and their enhancement in the full prestack data volume, which requires finite-offset (FO) processing. Finite-offset processing may provide improved resolution, especially in sparsely illuminated regions such as subsalt areas (Spinner et al., 2012), but due to the larger number of parameters, the problem is of higher dimensionality, which makes processing less stable and computationally more expensive than in the zero-offset (ZO) setting.

In order to combine the stability of zero-offset CRS processing (Jäger et al., 2001) with the improved illumination of finite-offset processing, we introduce a straightforward decomposition principle for diffractions. Based on the redundancy of zero-offset and finiteoffset information for diffractions, this new approach allows the direct prediction of finiteoffset diffraction attributes from zero-offset CRS processing results (Schwarz et al., 2014a; Bauer, 2014). In this fashion, the full prestack data volume is accurately characterized without carrying out computationally expensive generic finite-offset CRS processing (Zhang 
et al., 2001) or partial CRS stacking (Baykulov and Gajewski, 2009), where finite-offset traveltimes are extrapolated from zero-offset results.

[Figure 1 about here.]

\section{THEORY}

The common-reflection-surface (CRS) stack is a multi-parameter stacking technique, in which stacking is not only carried out along a traveltime moveout curve in offset direction, but along a traveltime moveout surface in both offset and midpoint direction (Jäger et al., 2001). CRS processing is purely data-driven and provides stacked sections with increased signal-to-noise ratio compared to the classical common-midpoint (CMP) stack, particularly in areas with complex geology and sparse illumination.

The CRS stack has been formulated for a central zero-offset (ZO) ray (Jäger et al., 2001) and for an arbitrary finite-offset (FO) central ray (Zhang et al., 2001). While the widely used zero-offset CRS stack is fast, stable and reasonably accurate for moderate lateral heterogeneity, finite-offset CRS shows its strengths in complex geological settings, where it provides improved resolution and illumination at the cost of being computationally more expensive and less stable (see, e.g., Spinner et al., 2012).

\section{Zero-offset common-reflection-surface}

In the reflection case, the $2 \mathrm{D}$ zero-offset CRS traveltime moveout depends on three parameters, the kinematic wavefield attributes $\alpha_{0}, R_{\mathrm{NIP}}$ and $R_{\mathrm{N}}($ Hubral, 1983). The latter ones, $R_{\mathrm{NIP}}$ and $R_{\mathrm{N}}$, are the radii of two ficticious wavefronts, the NIP-wavefront and the normal wavefront. While the NIP-wave stems from a ficticious point source placed on the reflector's 
point of normal incidence (NIP, see Figure 1(a)), the normal wave is emitted by a ficticious exploding reflector segment around the NIP. The angle $\alpha_{0}$ describes the direction, from which the two ficticious waves emerge at the central midpoint. For the computation of the traveltime moveout along the CRS, different formulations exist. In this work, the hyperbolic and the parabolic traveltime descriptions are used. The hyperbolic CRS traveltime (Jäger et al., 2001) reads

$$
t_{\mathrm{ZO}}^{2}\left(\Delta x_{m}, h\right)=\left(t_{0}^{\mathrm{ZO}}+\frac{2 \sin \alpha_{0}}{v_{0}} \Delta x_{m}\right)^{2}+\frac{2 t_{0} \cos ^{2} \alpha_{0}}{v_{0}}\left(\frac{\Delta x_{m}^{2}}{R_{\mathrm{N}}}+\frac{h^{2}}{R_{\mathrm{NIP}}}\right)
$$

where $\Delta x_{m}=x_{m}-x_{0}$ is the displacement from the central midpoint $x_{0}, h$ denotes the half-offset and $v_{0}$ the constant near-surface velocity. The parabolic CRS traveltime (Müller, 1999) is given by

$$
t_{\mathrm{ZO}}\left(\Delta x_{m}, h\right)=t_{0}^{\mathrm{ZO}}+\frac{2 \sin \alpha_{0}}{v_{0}} \Delta x_{m}+\frac{\cos ^{2} \alpha_{0}}{v_{0} R_{\mathrm{NIP}}} h^{2}+\frac{\cos ^{2} \alpha_{0}}{v_{0} R_{\mathrm{N}}} \Delta x_{m}^{2}
$$

In the special case of a diffraction, this traveltime moveout only depends on two parameters, because the NIP-wave and the normal wave coincide,

$$
t_{\mathrm{ZO}}\left(\Delta x_{m}, h\right)=t_{0}^{\mathrm{ZO}}+\frac{2 \sin \alpha_{0}}{v_{0}} \Delta x_{m}+\frac{\cos ^{2} \alpha_{0}}{v_{0} R_{\mathrm{NIP}}}\left(\Delta x_{m}^{2}+h^{2}\right)
$$


As illustrated in Figure 1(a), the NIP-wave approximates the actual physical wavefront of a diffraction in the zero-offset section $(h=0)$,

$$
t_{\mathrm{ZO}}\left(\Delta x_{m}\right)=t_{0}^{\mathrm{ZO}}+\frac{2 \sin \alpha_{0}}{v_{0}} \Delta x_{m}+\frac{\cos ^{2} \alpha_{0}}{v_{0} R_{\mathrm{NIP}}} \Delta x_{m}^{2}
$$

Based on the zero-offset CRS stack, Baykulov and Gajewski (2009) recently introduced the so-called partial CRS stack. In this method, finite-offset traces are simulated by application of zero-offset operators for local finite-offset stacks, which corresponds to the extrapolation of traveltimes from zero-offset. Figure 2(a) shows an example of a partial CRS stacking surface. The expansion point of the zero-offset operator is marked in red. Due to the smaller number of parameters this method is computationally more efficient than the generic finite-offset stack and in cases of moderate complexity and for small partial stacking apertures zero-offset operators perform reasonably well. However, the zero-offset approximation loses accuracy with increasing offsets and complexity. Finite-offset operators are more appropriate for local refinement at far offsets (see also Bauer, 2014; Schwarz et al., 2014a).

\section{Finite-offset common-reflection-surface}

The finite-offset CRS stack (Zhang et al., 2001) is a generalization of the zero-offset CRS stack to arbitrary source and receiver combinations, which allows the simulation of the full 
prestack data volume. Due to the higher dimensionality of the problem, the finite-offset CRS stack cannot be parametrized by only three parameters. Instead, because of the asymmetry of up- and downgoing raypaths, the finite-offset CRS parameters describe the attributes of the respective two-way wavefronts and a coupling coefficient. The five-parameter hyperbolic finite-offset CRS traveltime in source $\left(x_{s}=x_{m}-h\right)$ and receiver $\left(x_{g}=x_{m}+h\right)$ coordinates (Bergler et al., 2002) is given by

$$
\begin{aligned}
t_{\mathrm{FO}}^{2}\left(\Delta x_{s}, \Delta x_{g}\right)= & \left(t_{0}^{\mathrm{FO}}+\frac{\sin \alpha_{s}}{v_{s}} \Delta x_{s}+\frac{\sin \alpha_{g}}{v_{g}} \Delta x_{g}\right)^{2} \\
& +2 t_{0}^{\mathrm{FO}}\left(\frac{1}{2} B^{-1} A \Delta x_{s}^{2}+\frac{1}{2} D B^{-1} \Delta x_{g}^{2}+B^{-1} \Delta x_{s} \Delta x_{g}\right)
\end{aligned}
$$

where $\Delta x_{s}$ and $\Delta x_{g}$ denote the displacements from the central source and receiver pair $\left(x_{s}^{0}, x_{g}^{0}\right)$. Further, the angles of emergence at source and receiver are denoted by $\alpha_{s}$ and $\alpha_{g}{ }^{*}$ and accordingly, $v_{s}$ and $v_{g}$ are the near surface velocities at $x_{s}^{0}$ and $x_{g}^{0}$. The quantities $A, B$ and $D$ are the scalar elements of the surface-to-surface ray propagator matrix introduced by Bortfeld (1989). Zhang et al. (2001) describe these quantities in terms of the two emergence angles and three wavefront curvatures, which correspond to ficticious two-way waves observed in the common-shot (CS) and common-midpoint (CMP) configurations.

As it follows from a direct expansion of the traveltime, the parabolic finite-offset CRS traveltime formulation is physically more intuitive than its hyperbolic counterpart. Following Zhang et al. (2001) it reads

\footnotetext{
${ }^{*}$ Please note that we use a different sign convention for the emergence angles at source and receiver,
} which results in an alteration in signs compared to the original formula by Zhang et al. (2001) 


$$
\begin{aligned}
t_{\mathrm{FO}}\left(\Delta x_{s}, \Delta x_{g}\right)=t_{0}^{\mathrm{FO}} & +\frac{\sin \alpha_{s}}{v_{s}} \Delta x_{s}+\frac{1}{2} \frac{\cos ^{2} \alpha_{s}}{v_{s} R_{s}} \Delta x_{s}^{2} \\
& +\frac{\sin \alpha_{g}}{v_{g}} \Delta x_{g}+\frac{1}{2} \frac{\cos ^{2} \alpha_{g}}{v_{g} R_{g}} \Delta x_{g}^{2} \\
& +B^{-1} \Delta x_{s} \Delta x_{g}
\end{aligned}
$$

The coefficients of this second-order expression are parametrized by the emergence angles $\alpha_{s}, \alpha_{g}$ and the radii of curvature $R_{s}, R_{g}$ of the wavefronts measured in the common-shot (CS) and common-receiver (CR) configurations, respectively. The coefficient $B^{-1}$, which can be considered an expression of Snell's law, couples the moveouts in the CS and CR gathers. In the diffraction case, the coupling between the two-way wavefronts vanishes and they reduce to one-way waves (see Figure $1(\mathrm{~b})$ ). Hence, $B^{-1}$ becomes zero, which decouples the moveouts in the CS and CR configurations. Accordingly, the parabolic finite-offset response for diffractions reduces to

$$
\begin{aligned}
t_{\mathrm{FO}}\left(\Delta x_{s}, \Delta x_{g}\right)=t_{0}^{\mathrm{FO}} & +\frac{\sin \alpha_{s}}{v_{s}} \Delta x_{s}+\frac{1}{2} \frac{\cos ^{2} \alpha_{s}}{v_{s} R_{s}} \Delta x_{s}^{2} \\
& +\frac{\sin \alpha_{g}}{v_{g}} \Delta x_{g}+\frac{1}{2} \frac{\cos ^{2} \alpha_{g}}{v_{g} R_{g}} \Delta x_{g}^{2}
\end{aligned}
$$

[Figure 2 about here.] 


\section{Diffraction traveltime decomposition}

Due to the decoupling of diffraction raypaths, zero-offset and finite-offset information is redundant for diffractions (Schwarz et al., 2014a; Bauer et al., 2015): the CS and CR responses in a finite-offset measurement (see Figure 1(b)) are technically identical to two independent zero-offset measurements carried out at $x_{s}^{0}$ and $x_{g}^{0}$, as illustrated in Figure 1(c). Based on the assumption of reciprocity, which is valid for diffractions even in highly complex media, comparing equations (4) and (7) leads to the following system of equations (assuming a consistent choice of near-surface velocities):

$$
\begin{aligned}
& \alpha_{s}=\alpha_{0}^{s}, \\
& \alpha_{g}=\alpha_{0}^{g}, \\
& R_{s}=R_{\mathrm{NIP}}^{s}, \\
& R_{g}=R_{\mathrm{NIP}}^{g} .
\end{aligned}
$$

The superscripts $s$ and $g$ indicate the zero-offset attributes measured at the central source and receiver position, respectively (compare Figure 1(c)). This system of equations establishes the relations between zero-offset and finite-offset diffraction attributes. Also the finite-offset reference traveltime can be expressed by the two zero-offset reference traveltimes, 


$$
t_{0}^{\mathrm{FO}}=\frac{t_{0}^{\mathrm{ZO}, s}+t_{0}^{\mathrm{ZO}, g}}{2}
$$

Relations (8) and (9) indicate the redundancy of zero-offset and finite-offset information and thus allow the decomposition of any finite-offset operator into two independent zerooffset operators at a source $x_{s}^{0}$ and a receiver $x_{g}^{0}$ (Schwarz et al., 2014a),

$$
t_{\mathrm{FO}}\left(x_{s}^{0}, x_{g}^{0}, t_{0}^{\mathrm{FO}}, \alpha_{s}, \alpha_{g}, R_{s}, R_{g}\right)=\frac{t_{\mathrm{ZO}}\left(x_{s}^{0}, t_{0}^{\mathrm{ZO}, s}, \alpha_{0}^{s}, R_{\mathrm{NIP}}^{s}\right)}{2}+\frac{t_{\mathrm{ZO}}\left(x_{g}^{0}, t_{0}^{\mathrm{ZO}, g}, \alpha_{0}^{g}, R_{\mathrm{NIP}}^{g}\right)}{2}
$$

Since the traveltime decomposition principle is a fundamental property of diffractions, Equation (10) holds independently of the choice of zero-offset operators. If, e.g., hyperbolic operators such as (1) are used during the zero-offset processing, the finite-offset diffraction operators composed according to (10) correspond to double square root operators (Yilmaz, 2001), in which the two square roots are approximated independently.

Given the results from zero-offset processing are accurate, the introduced decomposition principle is exact for diffractions in arbitrary media and therefore we also refer to it as finite-offset prediction for diffractions. Figure 2(b) shows an example of a stacking surface constructed using the traveltime decomposition method. Note that this decomposition principle is only valid for diffractions, because it is based on the assumption of decoupled up- and downgoing raypaths. In the reflection case, the previously introduced equations do not hold. 


\section{APPLICATIONS}

\section{Diffraction traveltime fit}

In order to confirm the redundancy of zero-offset and finite-offset information, we carried out a diffraction traveltime fit on a simple synthetic model containing vertical and lateral heterogeneity by predicting finite-offset traveltimes and attributes solely based on a zerooffset fit and comparing the results to the generic finite-offset reference. The synthetic model consists of a plane interface at a depth of $300 \mathrm{~m}$ and a circular discontinuity with a radius of $5 \mathrm{~km}$, whose top point lies at a depth of $400 \mathrm{~m}$. The velocities are $2 \mathrm{~km} / \mathrm{s}$ above the plane interface, $3 \mathrm{~km} / \mathrm{s}$ below the interface and $3.5 \mathrm{~km} / \mathrm{s}$ inside the circular discontinuity. At a depth of $1 \mathrm{~km}$ and a lateral position of $5 \mathrm{~km}$ we placed a point diffractor. The model and the reference traveltimes were generated with the NORSAR raytracing software. The trav-

eltime fits were computed for each central source and receiver pair $\left(x_{s}^{0}, x_{g}^{0}\right)$ with MATLAB using a Nelder-Mead optimization scheme (Nelder and Mead, 1965) to find those wavefield attributes that minimize the traveltime error. For the computation of traveltime fitting surfaces in the zero-offset and finite-offset domain, the full parabolic traveltime formulations (2) and (6) were used.

[Figure 3 about here.]

Figures 3(a) and 3(b) show the obtained emergence angles at the source $\alpha_{s}$ for FO prediction and the generic finite-offset fit, respectively. The corresponding receiver attributes are not shown, since the general behavior is the same. Although it is only based on the results of a zero-offset traveltime fit, the traveltime decomposition approach almost perfectly reproduces the attributes which were obtained from a generic finite-offset fit performed for 
each finite-offset reference ray (corresponding to one sample of the plot). The symmetry in the results clearly shows the expected redundancy of zero-offset and finite-offset information for the diffraction case, which permits the prediction from zero-offset to finite-offset.

The traveltime errors of FO prediction and the generic finite-offset fit are displayed in Figures 3(c) and 3(d). Each sample of these plots shows the RMS traveltime error of the traveltime surface used for the fit at the corresponding central location. As expected from the theory, the misfit of FO prediction is identical to the one of the generic FO fit. This indicates that the introduced method is exact for diffractions, i.e., it is possible to obtain the exact finite-offset wavefield attributes by combining zero-offset attributes according to the introduced decomposition relations. The observed small errors can be explained by inaccuracies of the second-order traveltime approximation. Figure 4 shows the traveltime errors of FO prediction and the misfit resulting from global application of zero-offset operators, which corresponds to the partial CRS method. The results clearly reveal the advantages of the new method. Whereas the misfits coincide in the zero-offset section, partial CRS rapidly loses accuracy in offset direction due to the global application of zero-offset operators. However, the magnitude of the FO prediction error does not increase with offset, although both methods use exactly the same input.

Due to the significantly smaller number of traveltime fits, the traveltime decomposition approach only required $0.4 \%$ of the time the full finite-offset fitting needed to produce the same results in this study. Indeed, a challenging task of the traveltime decomposition is the matching of the two events stemming from the same diffractor at source and receiver, which is not required during traveltime fitting.

[Figure 4 about here.] 


\section{Implementation}

For the application to waveform data, we implemented the new diffraction traveltime decomposition approach into the zero-offset CRS workflow (Mann, 2002). As input, FO prediction requires the prestack data and results of the optimized zero-offset CRS stack, namely the optimized semblance and the attribute sections of the zero-offset wavefield attributes $\alpha_{0}$ and $R_{\mathrm{NIP}}$. In order to predict a finite-offset trace for the half-offset $h_{0}$ at the midpoint $x_{m}=x_{0}$, information from the locations $x_{s}^{0}=x_{0}-h_{0}$ and $x_{g}^{0}=x_{0}+h_{0}$ in the zero-offset section is required. At these locations, the two events which stem from the same diffractor have to be found and matched. In this implementation, different event combina-

tions are tested in order to find the one which provides the best fit. For each sample $t_{0}^{\mathrm{FO}}$ of the finite-offset trace, the source and receiver traces are searched for the corresponding diffraction events using relation (9). For the traveltime difference $\Delta t_{0}$ between two matching events the relation

$$
\Delta t_{0} \leq \frac{2 h_{0}}{v_{0}}
$$

holds and the event search may be confined. For each event pair found, the four finiteoffset wavefield attributes are extracted from the zero-offset attribute sections. They define the finite-offset diffraction operator for $\left(x_{0}, h_{0}, t_{0}^{\mathrm{FO}}\right)$. The parameters are tested for accuracy by computing the coherence for the constructed operator. In contrast to the generic finite-offset CRS stack, only the already estimated zero-offset attribute pairs at source and 
receiver need to be combined.

As output, FO prediction provides the finite-offset stacks, the respective semblance and the four finite-offset attribute sections for the predefined range of offsets. The method is able to handle an arbitrary number of conflicting events, if they are provided with the zero-offset results.

\section{Simple waveform example}

In order to verify the proposed method on waveform data, we applied it to a simple dataset based on the same velocity model as the diffraction traveltime fit, but this time containing an additional diffractor at a depth of $1.5 \mathrm{~km}$ and lateral position of $3.5 \mathrm{~km}$. The dataset contains a total of 401 CMPs and both the lateral extension of the seismic line and the maximum offset are $5 \mathrm{~km}$. Figure 5 compares various finite-offset semblance sections ranging from $500 \mathrm{~m}$ to $4000 \mathrm{~m}$ offset provided by FO prediction and the zero-offset-based partial CRS method (Baykulov and Gajewski, 2009). The results clearly reveal that the zero-offset operators used by partial CRS (Figure 5(b)) lose accuracy with increasing offset, because they extrapolate traveltimes. However, the FO prediction results (Figure 5(a)) are of very high quality along the whole offset range, because the decomposed finite-offset operators are accurate for arbitrary offsets. The decomposition relations directly connect zero-offset and finite-offset diffraction traveltimes and attributes and thus define the diffraction moveout globally, while the respective zero-offset-based operators are only accurate in local vicinities of each zero-offset reference ray.

[Figure 5 about here.] 


\section{Complex data example}

As a next step, we applied the diffraction traveltime decomposition approach to the synthetic Sigsbee 2A dataset. The key feature of this acoustic marine 2D model is a large homogeneous salt body, which is surrounded by sedimentary layers (see Figure 6). The rugged top of the salt body causes a significant amount of diffracted energy in the dataset. In addition, the model contains two horizontal lines of diffractors within the sedimentary layers to the left of and below the salt body and several fault structures. The dataset is modeled without a free surface, which is why it does not contain any surface-related multiple reflections. For the results provided in this work we used an excerpt of 400 CMPs taken from the complex part of the model (as indicated by the dotted lines in Figure 6), which contains both strong top of salt diffractions and diffracted energy from the subsalt area.

[Figure 6 about here.]

As in the previous examples, we applied partial CRS (Baykulov and Gajewski, 2009) and the new diffraction traveltime decomposition approach (FO prediction) to the data. The previous zero-offset CRS processing was carried out using a global optimization scheme for the attribute search (Walda and Gajewski, 2015) which accounted for a maximum of nine conflicting events at each sample. Both methods under investigation were applied with the same stacking apertures and processing parameters. Four different finite reference offsets ranging from $1 \mathrm{~km}$ to $7 \mathrm{~km}$ in steps of $2 \mathrm{~km}$ were considered. For a qualitative comparison of the emergence angle $\alpha_{s}$ to its generic reference we also applied full finite-offset CRS processing (Zhang et al., 2001) to the data.

[Figure 7 about here.] 
Figure 7 shows FO stacks of all four offsets for two different time ranges. The upper closeups (Figures 7(a) and 7(b)) are taken from 4 to $7 \mathrm{~s}$, i.e. the top of salt area. Whereas the traveltime decomposition approach allows us to image diffracted energy well up to the largest offset, partial CRS is only able to fit the strong top of salt diffractions at small offsets. Weaker events are hardly visible and with increasing offset all diffraction events become more blurry. Reflections, however, are still fitted with reasonable quality by partial CRS, whereas the traveltime decomposition inherently suppresses them due to the application of pure diffraction operators (compare the seafloor reflections visible at $5 \mathrm{~km}$ and $7 \mathrm{~km}$ offset). The closeups displayed below (Figures $7(\mathrm{c})$ and $7(\mathrm{~d})$ ), which were plotted with a smaller clip than the upper ones, are taken from 8 to $11 \mathrm{~s}$ and accordingly contain events stemming from the subsalt area. In general, we observe the same behavior as before: whereas the traveltime decomposition approach reveals a high imaging potential for diffractions throughout the whole offset range, the partial CRS stacks are of reasonable quality for small offsets, but become increasingly distorted at larger offsets, where the method fails to image weak events.

[Figure 8 about here.]

In order to evaluate the reliability of our result it is feasible to take into account the semblance coefficient, which serves as a good indicator of successful operator fit. Figure 8 displays the finite-offset semblance sections of the full time range of the dataset for the same four offset configurations as obtained by the new diffraction traveltime decomposition approach (left) and partial CRS (right). A comparison of the two images reveals the differences in the ability to find coherent energy in the data. Whereas our new approach images coherent diffracted energy throughout all offsets, partial CRS fails to accurately describe diffracted energy with increasing offset. Moreover, the total energy in the subsalt area 
is considerably higher for the prediction approach when compared to partial CRS, which should contribute to improved subsalt imaging. However, the reflections from the seafloor and the upper sedimentary layers are more coherent in the partial CRS results, whereas the traveltime decomposition inherently suppresses them. This effect becomes more apparent with increasing offset.

[Figure 9 about here.]

An advantage of the traveltime decomposition is that the new approach, unlike partial CRS, provides the four finite-offset diffraction attributes (emergence angle and NIP wavefront curvature at source and receiver, respectively) without any further optimization. Since the decomposition principle (10) is exact for diffractions, the attributes coincide with the respective estimates obtained from generic finite-offset processing. In this fashion, we can exploit the stability of zero-offset processing to obtain high-quality diffraction attributes for arbitrary offsets. To assess the quality and reliability of the obtained attributes we compare them to results from generic finite-offset CRS processing. Figure 9 exemplarily shows the emergence angle at the source $\alpha_{s}$ as obtained from the zero-offset-based diffraction traveltime decomposition (left) and from generic finite-offset CRS processing (right, Zhang et al., 2001 ) for the same data excerpt and offset configurations. Note that this is a qualitative comparison, because the algorithms differ and processing parameters cannot be chosen the same, which makes a quantitative comparison inequitable. Nevertheless, the results reveal the high quality of the emergence angle obtained from application of the new traveltime decomposition approach. The zero-offset-based attribute appears more stable and smooth, whereas the one provided by full finite-offset processing reveals stronger distortions, especially for weak events. A comparison of the attribute values of events, which are imaged by 
both methods, reveals that they coincide. This suggests that the decomposition principle is able to provide reliable prestack diffraction wavefield attributes, which may be used e.g. in the context of prestack stereotomography (Billette and Lambaré, 1998). Note that the angle values along the imaged reflections from sedimentary layers are only reliable if they are obtained from generic finite-offset processing, because the decomposition principle only works for diffractions.

\section{CONCLUSIONS AND OUTLOOK}

We have introduced a universal traveltime decomposition principle for diffractions, which is based on the decoupling of diffraction raypaths. As a result of this decoupling, the kinematic diffraction response is highly symmetric and thus, zero-offset and finite-offset information is redundant for diffractions. We found that every finite-offset diffraction operator can be decomposed into two independent zero-offset diffraction operators. In this fashion, we are able to image diffractions at arbitrary finite-offsets without loss of accuracy and solely based on zero-offset processing results. We proved the accuracy of the introduced relations by fitting diffraction traveltimes from heterogeneous media and comparing the results in terms of misfit and attributes to the finite-offset reference. In the context of the common-reflection-surface (CRS) stack (Jäger et al., 2001; Zhang et al., 2001), we used the traveltime decomposition principle to enhance prestack diffraction data and to obtain prestack diffraction wavefield attributes, which can be used for inversion. The results on simple waveform data showed the method's ability to fit diffractions at arbitrary offsets using only zero-offset attributes as input. Subsequent application to complex data confirmed the potential of the method for the enhancement of prestack diffraction data and attributes.

Since our method is entirely zero-offset based, it benefits from every improvement in the 
zero-offset processing. The use of zero-offset attributes obtained from a global optimization scheme with improved conflicting dip handling (Walda and Gajewski, 2015) has increased the quality of our results. Also, the application of non-hyperbolic operators such as i-CRS (Schwarz et al., 2014b) may lead to further improvement. Future work also includes the extension of the decomposition principle to 3D. For a point diffractor, this extension is straightforward. However, at structures such as edge or line diffractors, the decomposition principle is only applicable within that subset of directions, where the structure acts as a point diffractor. In addition, the unique properties of diffractions such as improved illumination and decoupling of raypaths are promising for the successful development of a diffraction-based tomographic scheme, which unites the efficiency of NIP-wave tomography (Duveneck, 2004) and the improved resolution of prestack stereotomography (Billette and Lambaré, 1998).

\section{ACKNOWLEDGMENTS}

This work was kindly supported by the sponsors of the Wave Inversion Technology (WIT) Consortium, Hamburg, Germany, and is part of the project Imaging steep structures with diffractions funded by the German Federal Ministry of Economic Affairs and Energy (BMWi 0325363C). For the generation of reference traveltimes, the NORSAR-3D raytracing software was used. The traveltime fit was carried out with MATLAB. The synthetic Sigsbee 2A dataset was provided by the Subsalt Multiples Attenuation and Reduction Technology Joint Venture (SMAART JV). 


\section{REFERENCES}

Bauer, A., 2014, From zero-offset to common-offset with diffractions: master's thesis, University of Hamburg.

Bauer, A., B. Schwarz, and D. Gajewski, 2015, Zero-offset based prediction of commonoffset diffraction traveltimes: Presented at the 77th EAGE Conference and Exhibition Workshops.

Baykulov, M., and D. Gajewski, 2009, Prestack seismic data enhancement with partial common-reflection-surface (CRS) stack: Geophysics, 74, V49-V58.

Bergler, S., E. Duveneck, G. Höcht, Y. Zhang, and P. Hubral, 2002, Common-reflectionsurface stack for converted waves: Studia geophysica et geodaetica, 46, 165-175.

Berkovitch, A., I. Belfer, Y. Hassin, and E. Landa, 2009, Diffraction imaging by multifocusing: Geophysics, 74, WCA75-WCA81.

Billette, F., and G. Lambaré, 1998, Velocity macro-model estimation from seismic reflection data by stereotomography: Geophysical Journal International, 135, 671-690.

Bortfeld, R., 1989, Geometrical ray theory: Rays and traveltimes in seismic systems (secondorder approximations of the traveltimes): Geophysics, 54, 342-349.

Dell, S., and D. Gajewski, 2011, Common-reflection-surface-based workflow for diffraction imaging: Geophysics, 76, S187-S195.

Duveneck, E., 2004, Velocity model estimation with data-derived wavefront attributes: Geophysics, 69, 265-274.

Fomel, S., E. Landa, and M. T. Taner, 2007, Poststack velocity analysis by separation and imaging of seismic diffractions: Geophysics, 72, U89-U94.

Hubral, P., 1983, Computing true amplitude reflections in a laterally inhomogeneous earth:

Geophysics, 48, 1051-1062. 
Jäger, R., J. Mann, G. Höcht, and P. Hubral, 2001, Common-reflection-surface stack: Image and attributes: Geophysics, 66, 97-109.

Khaidukov, V., E. Landa, and T. J. Moser, 2004, Diffraction imaging by focusing-defocusing: An outlook on seismic superresolution: Geophysics, 69, 1478-1490.

Mann, J., 2002, Extensions and applications of the common-reflection-surface stack method: PhD thesis, University of Karlsruhe.

Müller, T., 1999, The common reflection surface stack method - seismic imaging without explicit knowledge of the velocity model: PhD thesis, University of Karlsruhe.

Nelder, J., and R. Mead, 1965, A simplex method for function minimization: American Mathematical Monthly, 105, 523-528.

Schwarz, B., C. Vanelle, and D. Gajewski, 2014a, From zero-offset to common-offset with diffractions: Presented at the 76th EAGE Conference and Exhibition - Workshops.

Schwarz, B., C. Vanelle, D. Gajewski, and B. Kashtan, 2014b, Curvatures and inhomogeneities: an improved common-reflection-surface approach: Geophysics, 79, S231-S240.

Spinner, M., C. Tomas, P. Marchetti, C. Gallo, and S. Arfeen, 2012, Common-offset CRS for advanced imaging in complex geological settings: SEG Expanded Abstracts, doi: 10.1190/segam2012-1099.1.

Walda, J., and D. Gajewski, 2015, Common-reflection-surface stack improvement by differential evolution and conflicting dip processing: SEG Expanded Abstracts, doi: 10.1190/segam2015-5910957.1.

Yilmaz, O., 2001, Seismic data analysis: SEG, Tulsa.

Zhang, Y., S. Bergler, and P. Hubral, 2001, Common-reflection-surface (CRS) stack for common offset: Geophysical Prospecting, 49, 709-718. 


\section{LIST OF FIGURES}

1 In the diffraction case, the zero-offset NIP-wave (a) approximates the actual physical wavefront. In the finite-offset setting (b), the common-shot (CS) and common-receiver (CR) experiments may be resembled by two independent zero-offset NIP-wave experiments (c) at source $x_{s}^{0}$ and receiver $x_{g}^{0} . \ldots \ldots$

2 The partial CRS stacking surface (a) is based on global application of zerooffset operators. The FO prediction stacking surface (b) is based on a finiteoffset operator, which is constructed by two independent zero-offset diffraction operators at a source $x_{s}^{0}$ and a receiver $x_{g}^{0} \ldots \ldots \ldots$

3 Above: Emergence angles at the source $\alpha_{s}$ obtained from a) FO prediction and b) generic finite-offset fit. Below: Traveltime errors of c) FO prediction and d) generic finite-offset fit at the same scale. Each sample of the plots corresponds to the RMS traveltime error of one fit. . . . . . . . . . . .

4 Traveltime errors of a) FO prediction and b) global application of zero-offset operators plotted at the same scale. Each sample of the plots corresponds to the RMS traveltime error of one fit. . . . . . . . . . . . . . . . .

$5 \quad$ Finite-offset semblance sections of the simple waveform data for eight offsets from 500 (left) to $4000 \mathrm{~m}$ (right) generated by application of (a) the new diffraction traveltime decomposition approach and (b) the partial CRS method. 27

6 The Sigsbee 2 A velocity model. The dotted lines indicate the excerpt which was used for the results shown in this work. . . . . . . . . . . . . . .

$7 \quad$ Excerpts of finite-offset stacked sections of Sigsbee 2A for four offsets from 1000 (left) to $7000 \mathrm{~m}$ (right) from the top of salt area (above) and the subsalt area (below) generated by application of the new diffraction traveltime decomposition approach $(\mathrm{a}, \mathrm{c})$ and the partial CRS method $(\mathrm{b}, \mathrm{d}) \ldots \ldots$. .

$8 \quad$ Finite-offset semblance sections of Sigsbee 2A for four offsets from 1000 (left) to $7000 \mathrm{~m}$ (right) generated by application of (a) the new diffraction traveltime decomposition approach and (b) the partial CRS method. . . . . . . .

$9 \quad$ Finite-offset emergence angle at the source $\alpha_{s}\left[^{\circ}\right]$ of Sigsbee 2A for four offsets from 1000 (left) to $7000 \mathrm{~m}$ (right) generated by application of (a) the new diffraction traveltime decomposition approach and (b) generic finite-offset

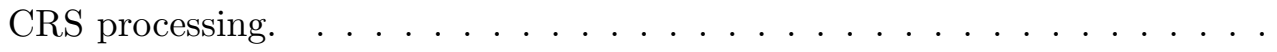




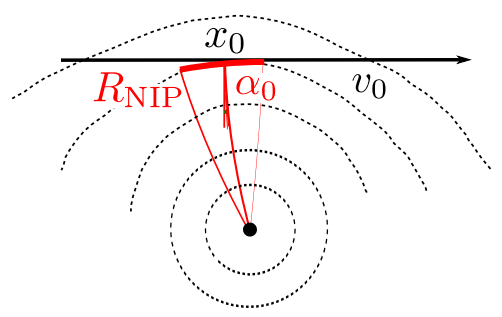

(a)

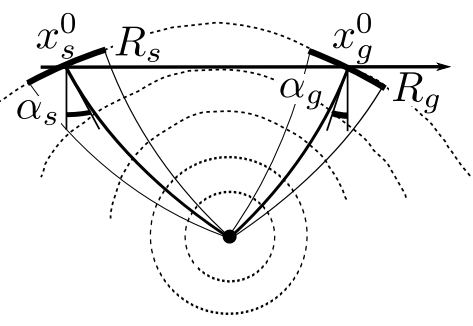

(b)

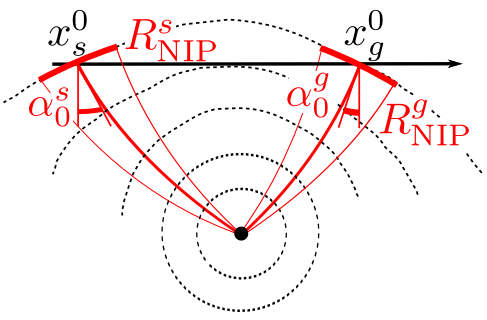

(c)

Figure 1: In the diffraction case, the zero-offset NIP-wave (a) approximates the actual physical wavefront. In the finite-offset setting (b), the common-shot (CS) and commonreceiver (CR) experiments may be resembled by two independent zero-offset NIP-wave experiments (c) at source $x_{s}^{0}$ and receiver $x_{g}^{0}$. 


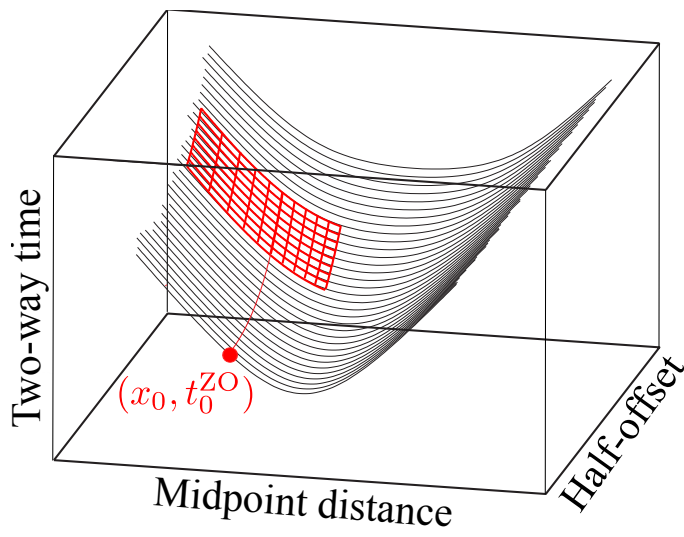

(a)

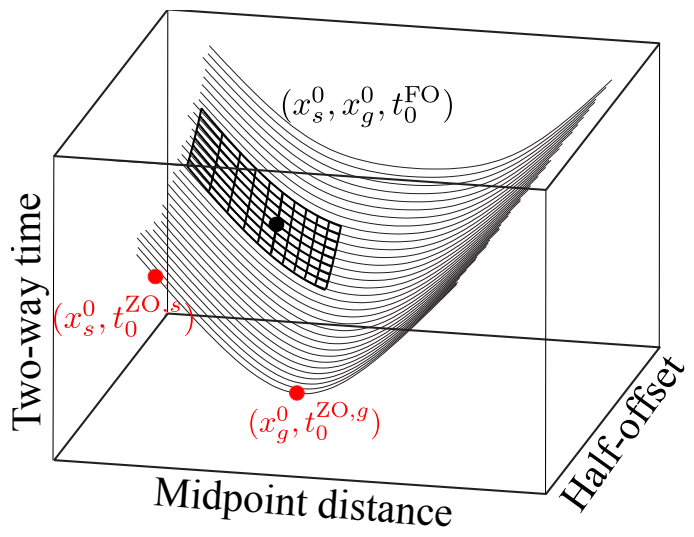

(b)

Figure 2: The partial CRS stacking surface (a) is based on global application of zero-offset operators. The FO prediction stacking surface (b) is based on a finite-offset operator, which is constructed by two independent zero-offset diffraction operators at a source $x_{s}^{0}$ and a receiver $x_{g}^{0}$. 


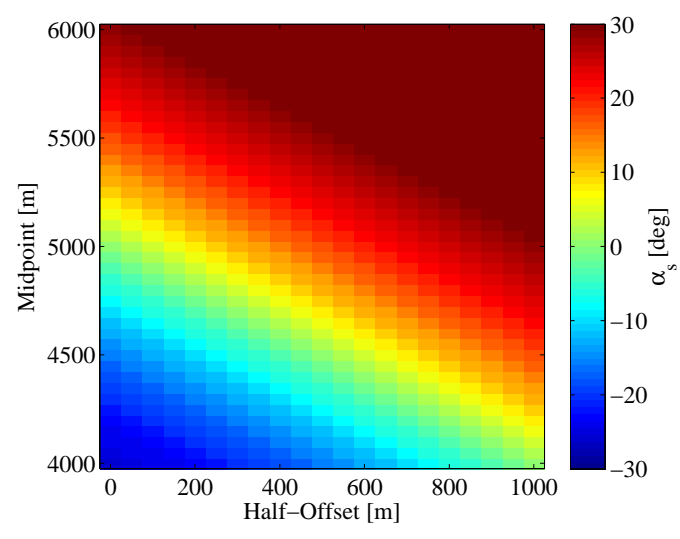

(a)

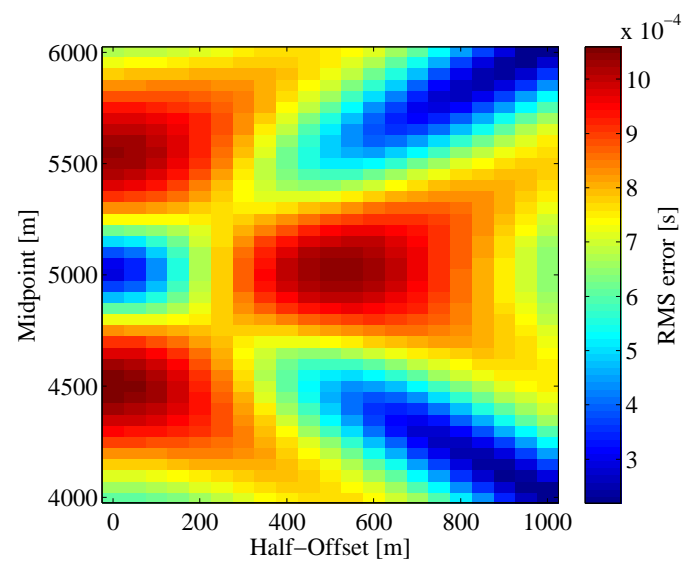

(c)

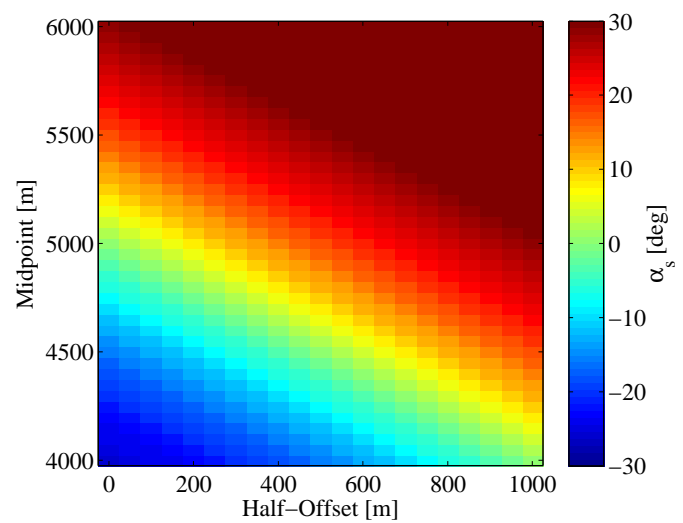

(b)

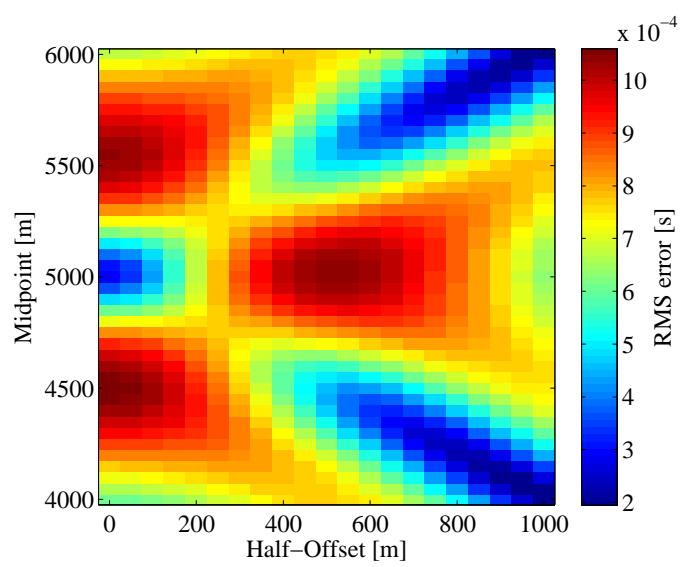

(d)

Figure 3: Above: Emergence angles at the source $\alpha_{s}$ obtained from a) FO prediction and b) generic finite-offset fit. Below: Traveltime errors of c) FO prediction and d) generic finiteoffset fit at the same scale. Each sample of the plots corresponds to the RMS traveltime error of one fit. 


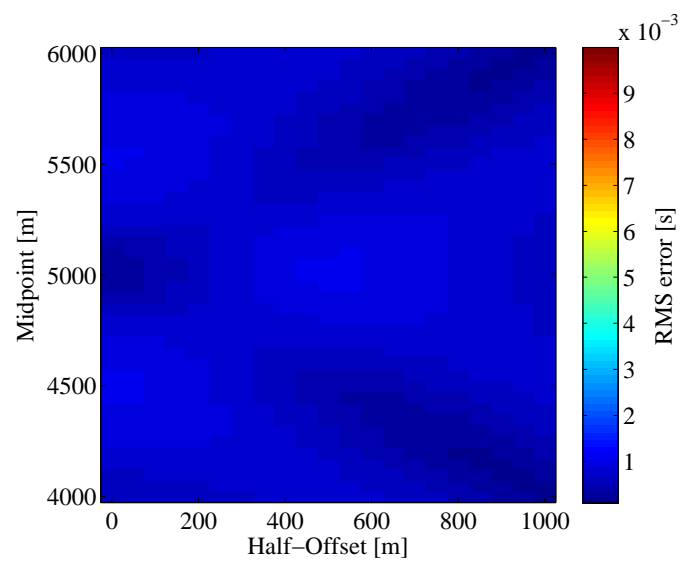

(a)

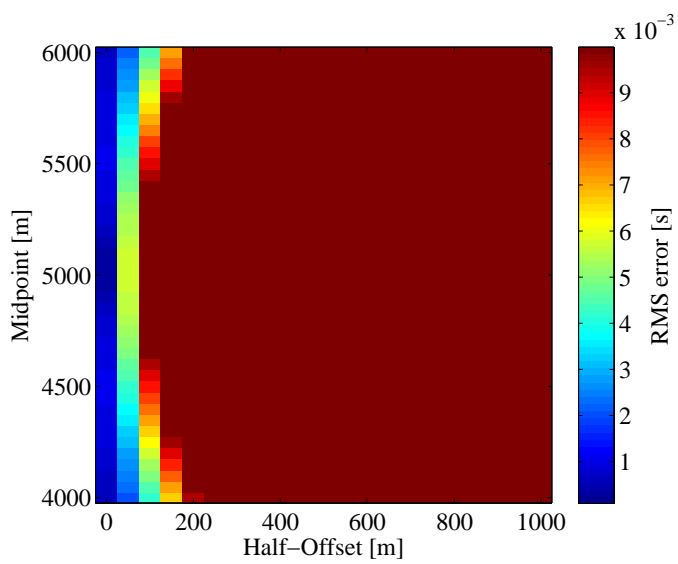

(b)

Figure 4: Traveltime errors of a) FO prediction and b) global application of zero-offset operators plotted at the same scale. Each sample of the plots corresponds to the RMS traveltime error of one fit. 


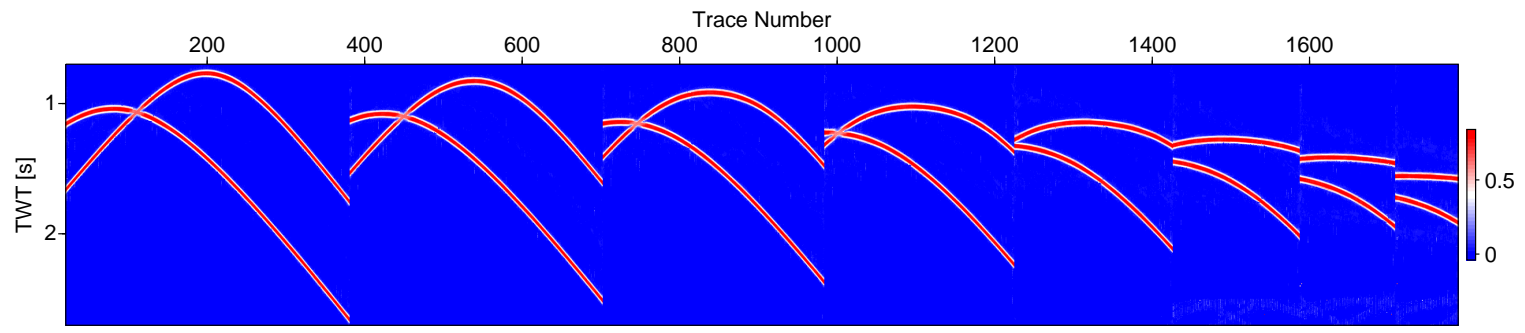

FO prediction semblance: offsets 500 - 4000 m

(a)

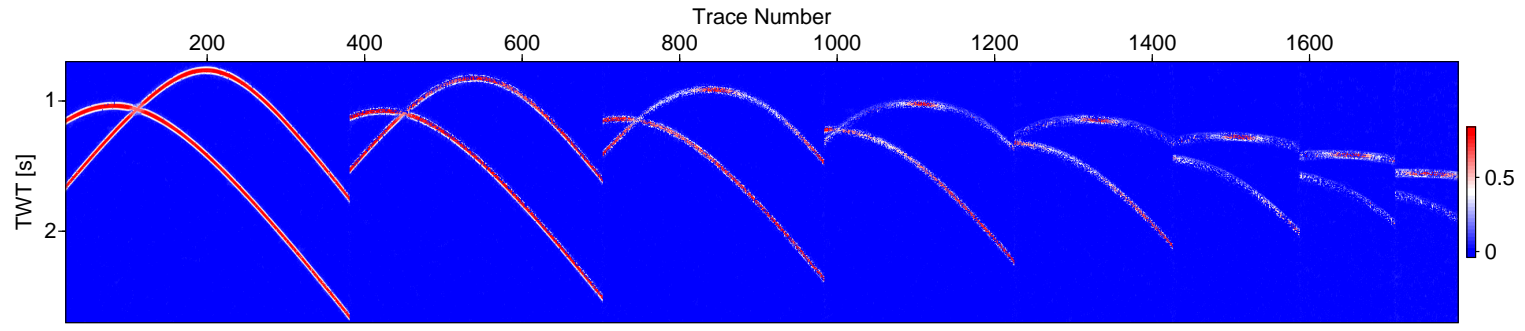

partial CRS semblance: offsets $500-4000$ m

(b)

Figure 5: Finite-offset semblance sections of the simple waveform data for eight offsets from 500 (left) to $4000 \mathrm{~m}$ (right) generated by application of (a) the new diffraction traveltime decomposition approach and (b) the partial CRS method. 


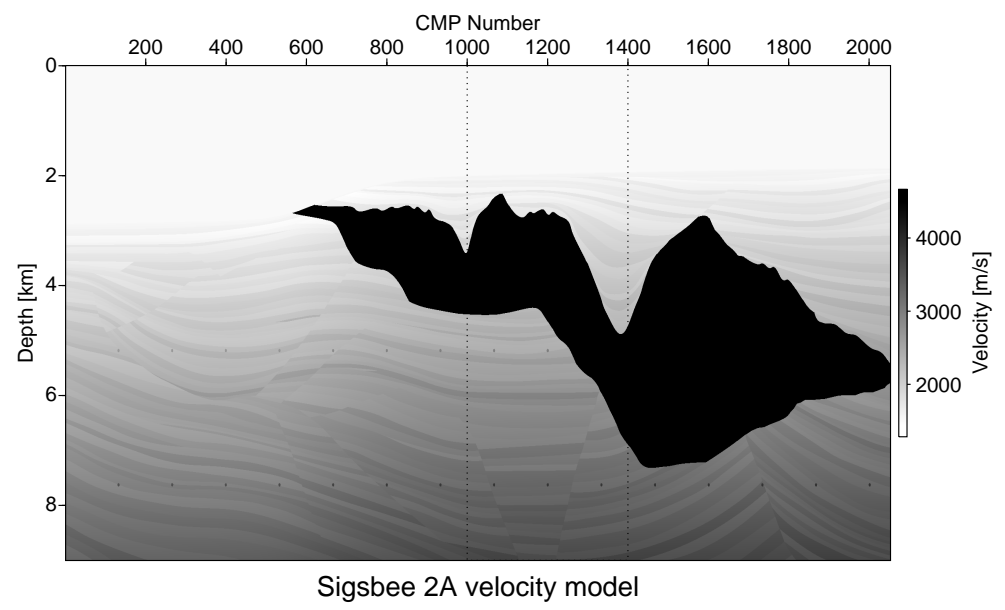

Figure 6: The Sigsbee 2A velocity model. The dotted lines indicate the excerpt which was used for the results shown in this work. 


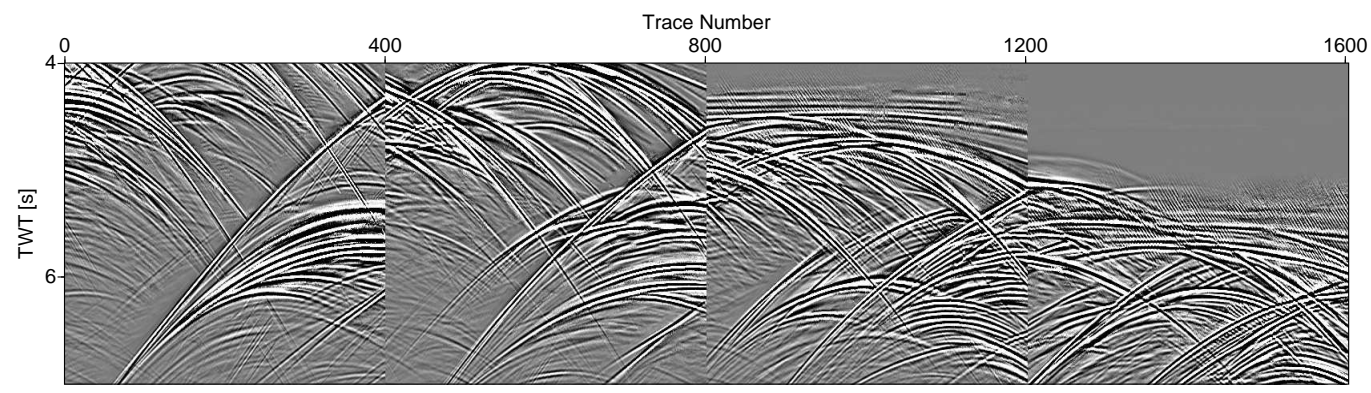

FO prediction: offsets $1000,3000,5000,7000 \mathrm{~m}$

(a)

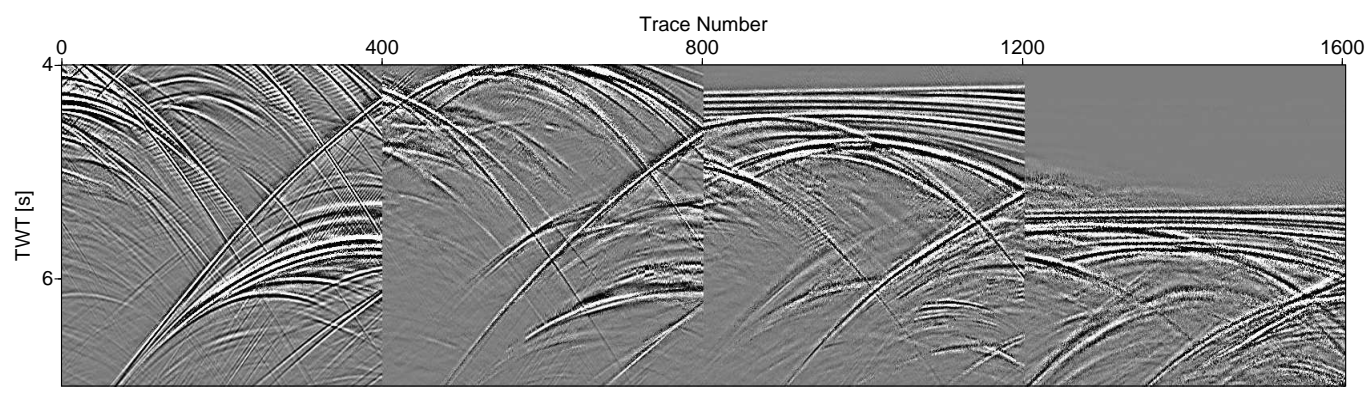

partial CRS: offsets $1000,3000,5000,7000 \mathrm{~m}$

(b)

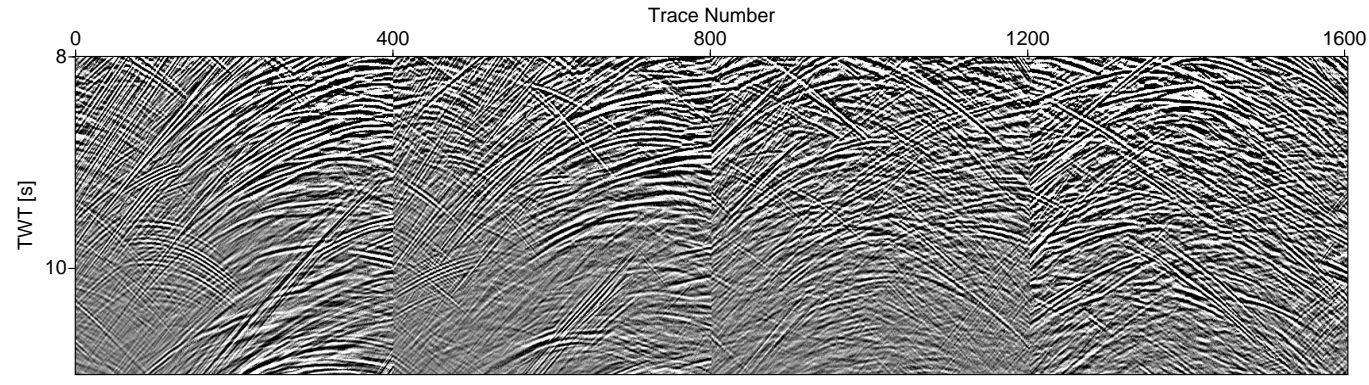

FO prediction: offsets $1000,3000,5000,7000 \mathrm{~m}$

(c)

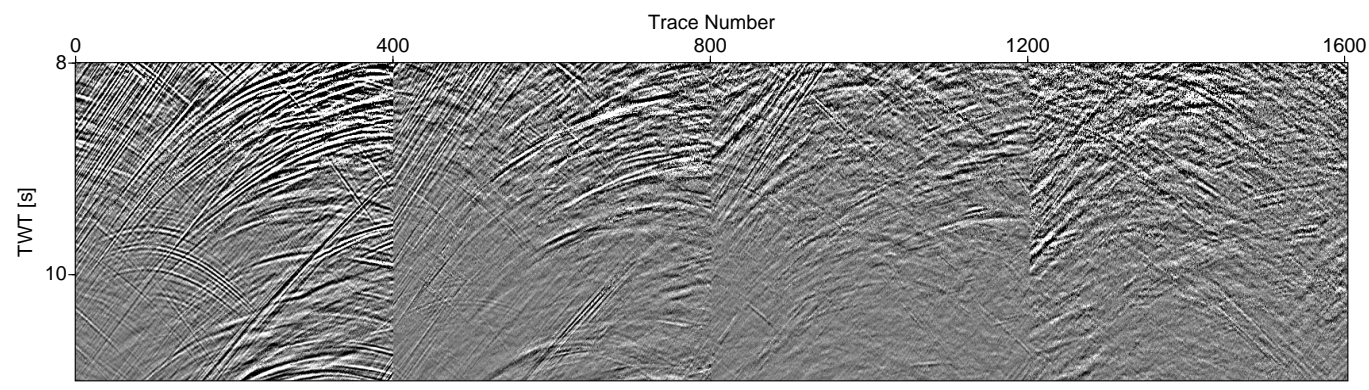

partial CRS: offsets $1000,3000,5000,7000 \mathrm{~m}$

(d)

Figure 7: Excerpts of finite-offset stacked sections of Sigsbee 2A for four offsets from 1000 (left) to $7000 \mathrm{~m}$ (right) from the top of salt area (above) and the subsalt area (below) generated by application of the new diffraction traveltime decomposition approach $(\mathrm{a}, \mathrm{c})$ and the partial CRS method $(b, d)$. 


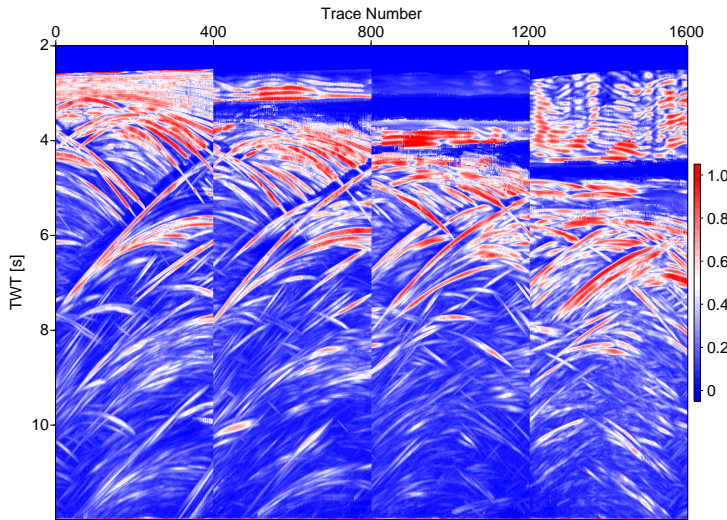

FO prediction: offsets $1000,3000,5000,7000 \mathrm{~m}$

(a)

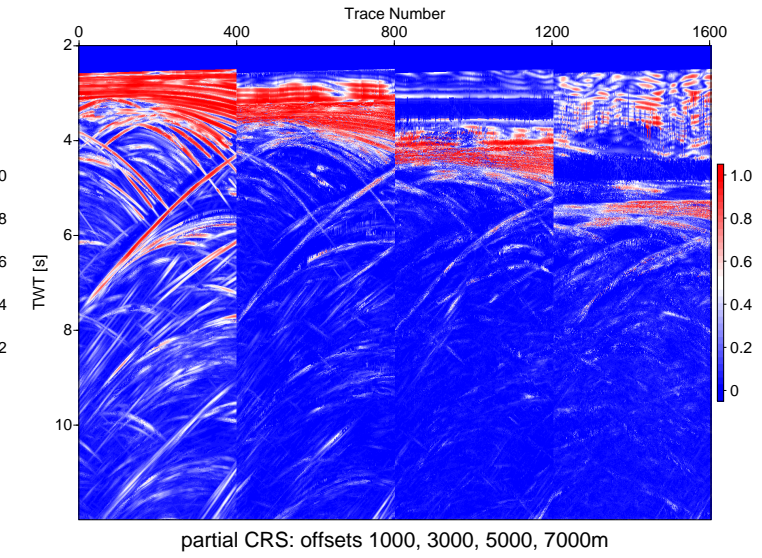

(b)

Figure 8: Finite-offset semblance sections of Sigsbee 2A for four offsets from 1000 (left) to $7000 \mathrm{~m}$ (right) generated by application of (a) the new diffraction traveltime decomposition approach and (b) the partial CRS method. 


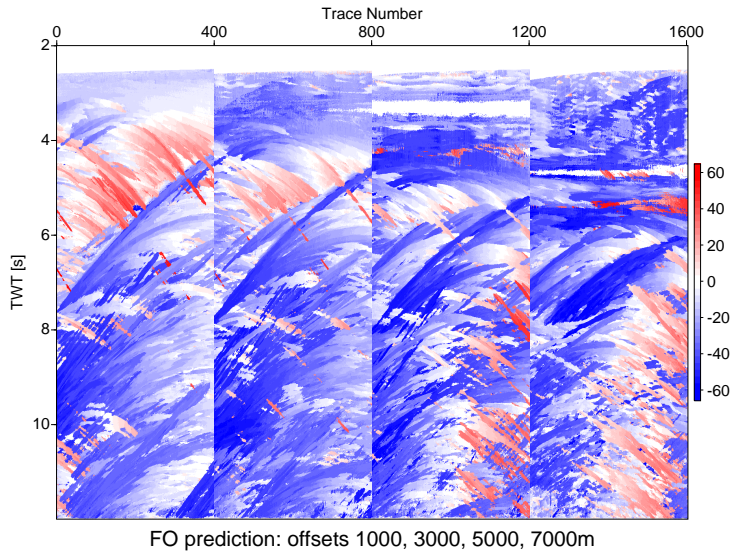

(a)

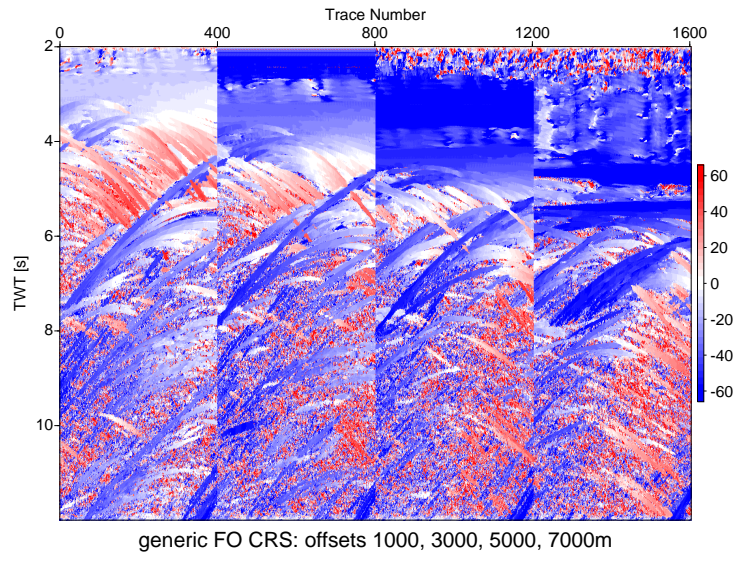

(b)

Figure 9: Finite-offset emergence angle at the source $\alpha_{s}\left[^{\circ}\right]$ of Sigsbee 2A for four offsets from 1000 (left) to $7000 \mathrm{~m}$ (right) generated by application of (a) the new diffraction traveltime decomposition approach and (b) generic finite-offset CRS processing. 